\title{
A FILTRATION OF WEYL MODULES FOR LARGE WEIGHTS
}

\author{
W. J. WONG \\ (Received 29 April 1986; revised 27 April 1987)
}

Communicated by H. Lausch

\begin{abstract}
A filtration is constructed for each dual Weyl module of a connected reductive group in prime characteristic $p$, and the quotients of the filtration are identified when the highest weight is far enough from the walls of the dominant chamber. The existence of certain composition factors is deduced.
\end{abstract}

1980 Mathematics subject classification (Amer. Math. Soc.): $20 \mathrm{G} 05$.

\section{Introduction}

Let $G$ be a connected reductive algebraic group over an algebraically closed field $F$ of prime characteristic $p$. Let $B$ be a Borel subgroup of $G, T$ a maximal torus of $B$, and suppose the roots of $(G, T)$ are ordered so that $B$ corresponds to the negative roots. If $\chi$ is a dominant character of $B$, the corresponding dual Weyl module of $G$ is the $G$-module $H^{0}(\chi)$ induced by $\chi$.

The most detailed information concerning the structure of $H^{0}(\chi)$ seems to have been obtained for "small" $\chi$, for example, when $\chi$ lies in the lowest $p^{2}$ alcove. In this paper we will obtain information when $\chi$ is large. For a given simple root $\alpha$, we construct a filtration of each $H^{0}(\chi)$, with terms corresponding to digits in the $p$-adic expansion of the inner product $\left(\chi, \alpha^{\nu}\right)$ of $\chi$ with the coroot $\alpha^{2}$ of $\alpha$ which are not equal to $p-1$. If $\chi$ is far enough from the walls of the dominant chamber which do not contain $\alpha$, the quotients of the filtration can be

(C) 1988 Australian Mathematical Society $0263-6115 / 88 \$ A 2.00+0.00$ 
identified as terms of the filtrations of modules $H^{0}(\lambda)$, for other characters $\lambda$ of $B$, linked to $\chi$ (Theorem 3.4). The existence of certain composition factors for $H^{0}(\chi)$ follows, and character formulas can be given for the terms of the filtration (Proposition 3.5).

The notation is standard. The character group $X(T)$ is denoted $X$, and the set of simple roots is denoted $S$. If $\alpha \in S$, the set of $\alpha$-dominant weights is

$$
X_{\alpha}^{+}=\left\{\chi \in X \mid\left(\chi, \alpha^{\nu}\right) \geq 0\right\}
$$

and the intersection of all the $X_{\alpha}^{+}$, for $\alpha$ in $S$, is the set $X^{+}$of dominant characters. The minimal parabolic subgroup corresponding to $\alpha$ is denoted $P_{\alpha}$.

All modules considered will be finite dimensional and rational. If $E$ is such a module for $B$, then $E$ induces a coherent sheaf on $G / B$ whose cohomology groups $H^{i}(E)$ are finite-dimensional rational $G$-modules. In particular, $H^{0}(E)$ is the $G$-module induced by $E$, and Frobenius reciprocity holds:

$$
\operatorname{Hom}_{G}\left(V, H^{0}(E)\right)=\operatorname{Hom}_{B}(V, E),
$$

where $V$ is any $G$-module. The same holds with $B$ replaced by any parabolic subgroup.

\section{Operations on $X_{\alpha}^{+}$}

For any non-negative integer $m$, we let $m_{k}$ denote the $k$ th digit of the $p$-adic expansion of $m$,

$$
m=\sum_{k \geq 0} m_{k} p^{k}, \quad 0 \leq m_{k} \leq p-1 .
$$

We shall need the following result of Andersen [1].

LEMMA 1.1. If $m, n$ are non-negative integers, then $\left(\begin{array}{c}m \\ n\end{array}\right) \equiv 0(\bmod p)$ if and only if $m_{k}<n_{k}$ for some $k$.

We choose a simple root $\alpha$, which will remain fixed throughout the paper. For each non-negative integer $k$, we define an operation $\tau_{k}$ on $X_{\alpha}^{+}$, by setting

$$
\tau_{k} \cdot \chi=\chi-\left(m_{k}+1\right) p^{k} \alpha, \quad \text { where } m=\left(\chi, \alpha^{\nu}\right) .
$$

Proceeding recursively, we can define

$$
\tau_{k}^{n} \cdot \chi=\tau_{k} \cdot\left(\tau_{k}^{n-1} \cdot \chi\right),
$$

provided $(n-1) p^{k} \leq m$ and $m_{k}<p-1$, or $2(n-1) p^{k} \leq m$ and $m_{k}=p-1$, these conditions being required to obtain $\tau_{k}^{n-1} \cdot \chi \in X_{\alpha}^{+}$. In particular, if $m<p^{k+1}$, then $\tau_{k} \cdot \chi \notin X_{\alpha}^{+}$, while if $m \geq p^{k+1}$ and $m_{k} \leq p-2$, then $\tau_{k} \cdot \chi \in X_{\alpha}^{+}$, and $p-2-m_{k}=n_{k}$, where $n=\left(\tau_{k} \cdot \chi, \alpha^{\curlyvee}\right)$. 
If $\chi \in X_{\alpha}^{+}, m=\left(\chi, \alpha^{\sim}\right)$, define

$$
J(\chi)=\left\{k \mid p^{k+1} \leq m, p-1>m_{k}\right\}
$$

the set of indices of the $p$-adic digits for $m$, below the leading one, which are not equal to $p-1$. From the above remarks we easily obtain

LEMMA 1.2. Let $\chi \in X_{\alpha}^{+}, k \in J(\chi)$. Then $\tau_{k} \cdot \chi \in X_{\alpha}^{+}$. Also, $k \in J\left(\tau_{k} \cdot \chi\right)$ if, and only if, $\tau_{k}^{2} \cdot \chi \in X_{\alpha}^{+}$. In particular, $\tau_{k}^{2} \cdot \chi \in X_{\alpha}^{+}$if $k$ is not the largest element of $J(\chi)$.

If $k \in J(\chi)$, then $\tau_{k} \cdot \chi$ lies in the orbit of $\chi$ under the affine Weyl group. In fact, $\tau_{k} \cdot \chi$ is "very strongly linked" to $\chi[4]$.

\section{2. $P_{\alpha}$-filtrations}

For $\chi$ in $X_{\alpha}^{+}$, let $H_{\alpha}^{0}(\chi)$ be the induced $P_{\alpha}$-module, that is, the group of global sections of the line bundle on $P_{\alpha} / B$ induced by the one-dimensional $B$-module of weight $\chi$. This has a unique irreducible submodule $M_{\alpha}(\chi)$. In this section we construct a filtration of $H_{\alpha}^{0}(\chi)$ which forms the basis for the filtrations of dual Weyl modules to be given in the next section.

The structure of $H_{\alpha}^{0}(\chi)$ is known explicitly [1]. Let $x_{\alpha}$ be an isomorphism from the additive group of $F$ to the root subgroup associated to $\alpha$, such that $t x_{\alpha}(z) t^{-1}=x_{\alpha}(\alpha(t) z)$, for $t$ in $T, z$ in $F$. If $m=\left(\chi, \alpha^{2}\right)$ then there is a basis $\left\{e_{b} \mid 0 \leq b \leq m\right\}$ of $H_{\alpha}^{0}(\chi)$, such that

$$
\begin{gathered}
t e_{b}=(\chi-b \alpha)(t) e_{b}, \\
x_{\alpha}(z) e_{b}=\sum_{a}\left(\begin{array}{l}
b \\
a
\end{array}\right) z^{b-a} e_{a} .
\end{gathered}
$$

The submodule $M_{\alpha}(\chi)$ is spanned by those $e_{b}$ for which $\left(\begin{array}{c}m \\ b\end{array}\right) \not \equiv 0(\bmod p)$, or, equivalently, $b_{k} \leq m_{k}$ for all $k$, by Lemma 1.1.

For each non-negative integer $j$, we let $K_{\alpha, j}(\chi)$ be the subspace spanned by those $e_{b}$ for which $b_{k} \leq m_{k}$, for all $k$ such that $0 \leq k<j$. Then

$$
H_{\alpha}^{0}(\chi)=K_{\alpha, 0}(\chi) \supseteq K_{\alpha, 1}(\chi) \supseteq K_{\alpha, 2}(\chi) \supseteq \cdots,
$$

and $K_{\alpha, j}(\chi)=M_{\alpha}(\chi)$ when $j$ is large.

PROPOSITION 2.1. Let $\chi \in X_{\alpha}^{+}$.

(a) $K_{\alpha, j}(\chi)$ is a submodule of $H_{\alpha}^{0}(\chi)$.

(b) If $j \notin J(\chi)$, then $K_{\alpha, j}(\chi)=K_{\alpha, j+1}(\chi)$.

(c) If $j \in J(\chi)$, then there is an exact sequence

$$
0 \rightarrow K_{\alpha, j+1}(\chi) \rightarrow K_{\alpha, j}(\chi) \rightarrow K_{\alpha, j+1}\left(\tau_{j} \cdot \chi\right) \rightarrow 0 .
$$


Proof. If $K_{\alpha, j}(\chi) \neq K_{\alpha, j+1}(\chi)$, then there exists an integer $b$ such that $0 \leq b \leq m$ and $b_{j}>m_{j}$. This implies that $j \in J(\chi)$, so that (b) holds.

Statement (a) holds when $j=0$. We assume that $K_{\alpha, j}(\chi)$ is a submodule, and proceed to show that $K_{\alpha, j+1}(\chi)$ is also a submodule. By (b), we may assume that $j \in J(\chi)$. We show in this case that there is a $P_{\alpha}$-homomorphism

$$
\theta: K_{\alpha, j}(\chi) \rightarrow H \alpha^{0}\left(\tau_{j} \cdot \chi\right)
$$

whose kernel is $K_{\alpha, j+1}(\chi)$.

The weights of $K_{\alpha, j}(\chi) / M_{\alpha}(\chi)$ are the characters $\chi-b \alpha$, where $0 \leq b \leq$ $m, b_{k} \leq m_{k}$ whenever $k<j$, but $b_{k}>m_{k}$ for some $k$. The least such number $b$ is $\left(m_{j}+1\right) p^{j}$. Thus the highest weight of $K_{\alpha, j}(\chi) / M_{\alpha}(\chi)$ is $\chi-\left(m_{j}+1\right) p^{j} \alpha=\tau_{j} \cdot \chi$. It follows that there exists a non-zero $B$-homomorphism of $K_{\alpha, j}(\chi)$ on the onedimensional module of weight $\tau_{j} \cdot \chi$. By Frobenius reciprocity, there exists a non-zero $P_{\alpha}$-homomorphism $\theta$ of $K_{\alpha, j}(\chi)$ into $H_{\alpha}^{0}\left(\tau_{j} \cdot \chi\right)$.

Let $q=\left(m_{j}+1\right) p^{j}, n=\left(\tau_{j} \cdot \chi, \alpha^{\swarrow}\right)=m-2 q$. We have a basis $\left\{e_{b} \mid 0 \leq b \leq m\right\}$ of $H_{\alpha}^{0}(\chi)$ as in (1), and a basis $\left\{f_{b} \mid 0 \leq b \leq n\right\}$ of $H_{\alpha}^{0}\left(\tau_{j} \cdot \chi\right)$, such that

$$
\begin{aligned}
t f_{b} & =(\chi-q \alpha-b \alpha)(t) f_{b}, \\
x_{\alpha}(z) f_{b} & =\sum_{a}\left(\begin{array}{l}
b \\
a
\end{array}\right) z^{b-a} f_{a},
\end{aligned}
$$

for $t$ in $T, z$ in $F$. Comparing weights, we see that, for $e_{b}$ in $K_{\alpha, j}(\chi)$,

$$
\theta\left(e_{b}\right)=c(b) f_{b-q},
$$

where $c(b) \in F$ (and $\theta\left(e_{b}\right)=0$, if $b<q$ or $b>m-q$ ). The action of $x_{\alpha}(z)$ shows that

$$
\sum_{a}\left(\begin{array}{l}
b \\
a
\end{array}\right) z^{b-a} c(a) f_{a-q}=c(b) \sum_{a}\left(\begin{array}{c}
b-q \\
a
\end{array}\right) z^{b-q-a} f_{a} .
$$

Comparing coefficients of $f_{0}$, we see that

$$
c(b)=\left(\begin{array}{l}
b \\
q
\end{array}\right) c(q) \text {. }
$$

Since $\theta \neq 0, c(q) \neq 0$. We see that the kernel of $\theta$ is spanned by those $e_{b}$ for which $b_{k} \leq m_{k}$ for all $k<j$, and $\left(\begin{array}{l}b \\ q\end{array}\right) \equiv 0(\bmod p)$. By Lemma 1.1, the latter condition is equivalent to $b_{j} \leq m_{j}$. Hence, the kernel of $\theta$ is $K_{\alpha, j+1}(\chi)$, which is therefore a submodule. This proves (a) by induction.

Also, the image of $\theta$ is the subspace of $H_{\alpha}^{0}\left(\tau_{j} \cdot \chi\right)$ spanned by all the $f_{b-q}$ such that $b_{k} \leq m_{k}$ for all $k<j$, and $b_{j} \geq m_{j}+1$. Since $n_{j}=p-2-m_{j}$, it is easy to check that this is equivalent to requiring that the number $a=b-q$ satisfies the condition that $a_{k} \leq n_{k}$, for all $k \leq j$. Hence the image of $\theta$ is $K_{\alpha, j+1}(\chi)$, and (c) holds. This proves the proposition. 
If the repetitions given in (b) are eliminated, we have found a filtration for $H_{\alpha}^{0}(\chi)$, whose quotients are given by terms of similar filtrations for modules $H_{\alpha}^{0}\left(\tau_{j} \cdot \chi\right)$ corresponding to weights $\tau_{j} \cdot \chi$ linked to $\chi$.

It is natural to try to compare our filtration with the Jantzen filtration. In [2], Jantzen defined a filtration of Weyl modules in terms of contravariant forms. For the dual Weyl module $H_{\alpha}^{0}(\chi)$ of $P_{\alpha}$, this is the series

$$
M_{\alpha}(\chi)=V_{1} \subset V_{2} \subset \cdots \subset V_{n-j(1)} \subset V_{n-j(1)+1}=H_{\alpha}^{0}(\chi),
$$

where $j(1)$ is the smallest member of $J(\chi)\left(j(1)=n\right.$ if $J(\chi)$ is empty), and $V_{k}$ is the subspace spanned by the elements $e_{b}$ for which $\left(\begin{array}{c}m \\ b\end{array}\right)$ is not divisible by $p^{k}$. The filtration $\left\{K_{\alpha, j}(\chi)\right\}$ is in general coarser than Jantzen's filtration. It has $|J(\chi)|+1$ distinct terms, and $|J(\chi)|+1 \leq n-j(1)+1$. The distribution of the $K_{\alpha, j}(\chi)$ along Jantzen's filtration is indicated by a calculation showing that

$$
V_{n-j+1} \supseteq K_{\alpha, j}(\chi), \quad V_{n-j} \nsupseteq K_{\alpha, j}(\chi),
$$

for $j$ in $J(\chi)$. The connection between the two filtrations is, however, not very close. If $J(\chi)$ is nonempty, the largest proper submodule in the filtration $\left\{K_{\alpha, j}(\chi)\right\}$ is $K_{\alpha, j(1)+1}(\chi)$. If $m_{j(1)+1}>0$, then it can be shown that this submodule does not include $V_{2}$, the smallest submodule of the Jantzen filtration above the unique simple submodule $M_{\alpha}(\chi)$.

\section{3. $G$-filtrations}

If $E$ is a $B$-module (or $P_{\alpha}$-module), then $E$ induces a sheaf on $G / B$ (or $G / P_{\alpha}$ ), whose cohomology groups are $G$-modules, denoted $H^{i}(E)$. In particular, if $\chi \in X^{+}$and we denote the corresponding one-dimensional $B$-module also as $\chi$, then $H^{0}(\chi)$ is called a dual Weyl module, and has the irreducible module $M(\chi)$ of highest weight $\chi$ as its unique minimal submodule.

We use the filtration of $H_{\alpha}^{0}(\chi)$ given in the last section to construct a filtration of $H^{0}(\chi)$ in the obvious way.

Proposition 3.1. Let $\chi \in X^{+}$, and let $K_{j}(\chi)=H^{0}\left(K_{\alpha, j}(\chi)\right)$. Then,

$$
H^{0}(\chi)=K_{0}(\chi) \supseteq K_{1}(\chi) \supseteq K_{2}(\chi) \supseteq \cdots \supseteq M(\chi) .
$$

ProOF. By [1], $K_{0}(\chi)=H^{0}\left(H_{\alpha}^{0}(\chi)\right)=H^{0}(\chi) \supseteq M(\chi)$. Assume that $K_{j}(\chi) \supseteq M(\chi)$. If $j \in J(\chi)$, then, by Proposition 2.1 (c), we have an exact sequence

$$
0 \rightarrow K_{j+1}(\chi) \rightarrow K_{j}(\chi) \rightarrow K_{j+1}\left(\tau_{j} \cdot \chi\right) .
$$

Since $K_{j+1}\left(\tau_{j} \cdot \chi\right)$ is contained in $H^{0}\left(\tau_{j} \cdot \chi\right)$, whose highest weight $\tau_{j} \cdot \chi$ is less than $\chi, M(\chi)$ does not occur in $K_{j+1}\left(\tau_{j} \cdot \chi\right)$. Hence $K_{j+1}(\chi) \supseteq M(\chi)$. The 
same holds trivially if $j \notin J(\chi)$. By induction, $K_{j}(\chi) \supseteq M(\chi)$, for all $j$. This proves the proposition.

Eliminating repetitions $K_{j}(\chi)=K_{j+1}(\chi)$ when $j \notin J(\chi)$, we obtain a filtration of $H^{0}(\chi)$. We would like to identify the quotient modules in this filtration, as in the case of the filtration of $H_{\alpha}^{0}(\chi)$. This can be done if $\chi$ is far enough from the walls of the fundamental Weyl chamber which do not contain $\alpha$.

LEMMA 3.2. Let $\chi \in X^{+}, j \geq 0$. If $\chi$ satisfies the condition

$$
C(j):\left(\chi, \beta^{\curlyvee}\right) \geq\left[\frac{N}{2}\right]\left(-\left(\alpha, \beta^{\curlyvee}\right)\right) \sum\left\{p^{k+1} \mid k \in J(\chi), k<j\right\}, \text { all } \beta \text { in } S,
$$

where $N$ is the number of positive roots, then $H^{i}\left(K_{\alpha, j}(\chi)\right)=0$, for all $i>0$.

ProOF. We remark that in the condition $C(j)$ we need to consider only those $\beta$ which are different from $\alpha$ and not orthogonal to $\alpha$. There are at most three such roots $\beta$, and $-3 \leq\left(\alpha, \beta^{\nu}\right)<0$ for these $\beta$. Note also that, if $\chi$ satisfies $C(j)$ and $j \in J(\chi)$, then $\tau_{j} \cdot \chi \in X^{+}$, by Lemma 1.2 , and $\tau_{j} \cdot \chi$ also satisfies $C(j)$.

If $j=0$, the result holds, by Kempf's vanishing theorem [3], since $H^{i}\left(H_{\alpha}^{0}(\chi)\right)$ $=H^{i}(\chi)$, by [1]. We assume the result holds for $j$, and prove that it holds for $j+1$.

If $\mu \in X^{+}, \mu$ satisfies $C(j)$ and $j \in J(\mu)$, then the exact sequence of Proposition 2.1 (c) and the vanishing of $H^{i}\left(K_{\alpha, j}(\mu)\right)$ give an isomorphism

$$
H^{i}\left(K_{\alpha, j+1}\left(\tau_{j} \cdot \mu\right)\right) \simeq H^{i+1}\left(K_{\alpha, j+1}(\mu)\right), \quad i>0 .
$$

This argument can be repeated, using Lemma 1.2, to obtain

$$
H^{i}\left(K_{\alpha, j+1}\left(\tau_{j}^{n} \cdot \mu\right)\right) \simeq H^{i+n}\left(K_{\alpha, j+1}(\mu)\right),
$$

if $i>0, \tau_{j}^{n} \cdot \mu \in X^{+}$.

Now suppose that $\chi \in X^{+}$, and that $\chi$ satisfies $C(j+1)$. If $j \notin J(\chi)$, then $H^{i}\left(K_{\alpha, j+1}(\chi)\right)=H^{i}\left(K_{\alpha, j}(\chi)\right)=0$ for $i>0$, since $\chi$ satisfies $C(j)$. Assume $j \in J(\chi)$. Let $r=\left[\frac{N}{2}\right]$, and set

$$
\mu=\chi+r p^{j+1} \alpha .
$$

Using the condition $C(j+1)$, we see that $\mu \in X^{+}$and $\mu$ satisfies $C(j)$. Since $\chi=\tau_{j}^{2 r} \cdot \mu$, the result of the last paragraph shows that, for $i>0$,

$$
H^{i}\left(K_{\alpha, j+1}(\chi)\right) \simeq H^{i+2 r}\left(K_{\alpha, j+1}(\mu)\right) .
$$

Since $i+2 r \geq N$, and $G / P_{\alpha}$ has dimension $N-1$, the right side is 0 . Thus the result holds for $j+1$. By induction, the lemma is proved. 
PROPOSITION 3.3. Let $\chi \in X^{+}, j \geq 0$, and suppose $\chi$ satisfies the condition $C(j+1)$. If $j \notin J(\chi)$, then $K_{j}(\chi)=K_{j+1}(\chi)$. If $j \in J(\chi)$, then there exists an exact sequence

$$
0 \rightarrow K_{j+1}(\chi) \rightarrow K_{j}(\chi) \rightarrow K_{j+1}\left(\tau_{j} \cdot \chi\right) \rightarrow 0
$$

PROOF. This follows from Proposition 2.1 and the vanishing of $H^{1}\left(K_{\alpha, j+1}(\chi)\right)$ given by Lemma 3.2.

If $j \in J(\chi)$, and $k$ is the least element of $J(\chi)$ greater than $j$, then $K_{j+1}(\chi)=$ $K_{k}(\chi)$. We can rephrase Proposition 3.3 in the following way. Arrange the elements of $J(\chi)$ in ascending order,

$$
J(\chi)=\{j(1), j(2), \ldots, j(n)\}, \quad j(1)<j(2)<\cdots<j(n) .
$$

For $i \geq 0$, define

$$
\begin{array}{r}
L_{i}(\chi)=K_{j(i+1)}(\chi), \quad \text { if } i \leq n-1, \\
L_{\mathbf{i}}(\chi)=K_{j(n)+1}(\chi), \quad \text { if } i \geq n .
\end{array}
$$

Then, for $i \leq n, L_{i}(\chi)=K_{j(i)+1}(\chi)$. Note that $j(i)$ is the $i$ th element of $J\left(\tau_{j(i)} \cdot \chi\right)$, unless possibly if $i=n$, by Lemma 1.2 , and in any case

$$
L_{i}\left(\tau_{j(i)} \cdot \chi\right)=K_{j(i)+1}\left(\tau_{j(i)} \cdot \chi\right) .
$$

We obtain the following restatement of Proposition 3.3.

THEOREM 3.4. Let $\chi \in X^{+}$, and let the elements of $J(\chi)$ be $j(1), j(2), \ldots$, $j(n)$ in ascending order. Suppose that, for some value of $r$ such that $r \leq n$,

$$
\left(\chi, \beta^{\sim}\right) \geq\left[\frac{N}{2}\right]\left(-\left(\alpha, \beta^{\sim}\right)\right) \sum_{i \leq r} p^{j(i)+1},
$$

for all $\beta$ in $S$. Then the filtration

$$
H^{0}(\chi)=L_{0}(\chi) \supset L_{1}(\chi) \supset \cdots \supset L_{r}(\chi)
$$

has quotients given by

$$
L_{i-1}(\chi) / L_{i}(\chi) \simeq L_{i}\left(\tau_{j(i)} \cdot \chi\right), \quad 1 \leq i \leq r .
$$

In particular, $M\left(\tau_{j(i)} \cdot \chi\right)$ is a composition factor of $H^{0}(\chi)$, for $1 \leq i \leq r$.

The last statement follows from the fact that $L_{i}\left(\tau_{j(i)} \cdot \chi\right) \supseteq M\left(\tau_{j(i)} \cdot \chi\right)$, by Proposition 3.1.

From Theorem 3.4, we obtain exact sequences

$$
0 \rightarrow L_{i}(\chi) \rightarrow L_{i-1}(\chi) \rightarrow L_{i-1}\left(\tau_{j(i)} \cdot \chi\right) \rightarrow \cdots \rightarrow L_{i-1}\left(\tau_{j(i)}^{m(i)} \cdot \chi\right) \rightarrow 0,
$$


where $m(i)$ is the largest integer such that $\tau_{j(i)}^{m(i)} \cdot \chi \in X^{+}$. These enable us to write down formulas for the characters of the $L_{i}(\chi)$. If $\lambda \in X^{+}$, let $\operatorname{ch} \lambda$ be the corresponding formal character of $H^{0}(\lambda)$, given by Weyl's formula. Note that, if $\tau_{j(i)}^{k} \cdot \chi \in X^{+}$, then $j(1), j(2), \ldots, j(i-1)$ are the first $i-1$ elements of $J\left(\tau_{j(i)}^{k} \cdot \chi\right)$. An easy induction now gives the desired formula.

PROPOSITION 3.5. In Theorem 3.4, the character of $L_{i}(\chi)$ is equal to

$$
\sum(-1)^{a(1)+a(2)+\cdots+a(i)} \operatorname{ch}\left(\tau_{j(1)}^{a(1)} \tau_{j(2)}^{a(2)} \ldots \tau_{j(i)}^{a(i)} \cdot \chi\right),
$$

where the sum is taken over all $i$-tuples $(a(1), a(2), \ldots a(i))$ of non-negative integers, such that $\tau_{j(1)}^{a(1)} \tau_{j(2)}^{a(2)} \ldots \tau_{j(i)}^{a(i)} \cdot \chi \in X^{+}$.

There appears to be no close connection between the filtration of Proposition 3.1 and Jantzen's filtration for $H^{0}(\chi)$ [2], and the character formula of Proposition 3.5 does not seem to be related to Jantzen's sum formula for the terms of his filtration.

\section{References}

[1] H. H. Andersen, 'The first cohomology group of a line bundle on $G / B$ ', Invent. Math. 51 (1979), 287-296.

[2] J. C. Jantzen, 'Weyl modules for groups of Lie type', Proceedings of the London Math. Soc. Research Symposium on Finite Simple Groups, Durham, 1978.

[3] G. Kempf, 'Linear systems on homogeneous spaces', Ann. of Math. 103 (1976), 557-591.

[4] W. J. Wong, 'Very strong linkage for cohomology groups of line bundles on $G / B$ ', $J$. Algebra, to appear.

Department of Mathematics

University of Notre Dame

Notre Dame, Indiana 46556

U.S.A 